\title{
Simulation of QCD and other similar theories
}

\author{
I. Montvay ${ }^{a}$

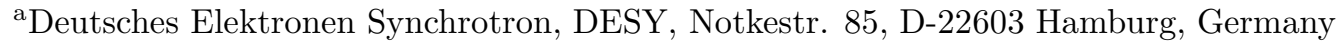

In QCD one can change the representation of the gauge group for quarks and/or the gauge group itself. Examples of such generalizations are: (a) supersymmetric Yang-Mills theory with gauge group SU(2) or SU(3); (b) QCD with $\mathrm{SU}(2)$ colour and quarks in the adjoint representation; (c) QCD with three equal-mass quarks. Experience and perspectives in simulations of these theories by applying the two-step multi-bosonic algorithm is described. As an example of an interesting physical problem in these theories the expected spontaneous CPviolation in $\mathrm{QCD}$ with three equal-mass quarks is discussed.

\section{INTRODUCTION}

Quantum chromodynamics is most probably the correct theory of strong interactions. It belongs to a class of relativistic quantum field theories in four dimensions which are believed to describe a wide range of physical phenomena consistently from zero to infinite energies.

Examples of generalizations of QCD are given in the abstracts. Numerical simulations of these theories can give important non-perturbative insight in low energy dynamical features as confinement and chiral symmetry breaking. Restricting numerical studies to the quenched approximation is obviously unsatisfactory. In QCD, besides dynamical simulations with two equal mass quarks, the investigation of $\mathbf{2} \oplus \mathbf{1}$ light dynamical quark flavours is very important because it is a good approximation of the physically relevant $u_{-}, d-$ and $s$-quark system.

A fermion simulation algorithm which is able to deal with QCD-like theories for small fermion masses in sufficiently large volumes is the twostep multi-bosonic (TSMB) algorithm [1,2]. In a recent large-scale simulation [3 5] this algorithm has been used for the numerical study of supersymmetric Yang-Mills theories which contain "gauginos" i.e. Majorana fermions in the adjoint representation of the gauge group. The algorithm could cope with a potentially difficult situation where massless gauginos are playing an important dynamical rôle in reasonably large physical volumes. It is an interesting question whether this experience can be extended, for instance, to QCD with $\mathbf{2} \oplus \mathbf{1}$ light dynamical quarks.

\section{FERMION ALGORITHM}

The TSMB algorithm is based on the multibosonic representation of the fermion determinant [6]:

$$
|\operatorname{det}(Q)|^{N_{f}} \simeq \frac{1}{\operatorname{det} P_{n}\left(Q^{\dagger} Q\right)} .
$$

Here $Q$ is the fermion matrix and $N_{f}$ degenerate flavours are considered. The polynomial $P_{n}(x)$ is an approximation of the function $x^{-\alpha},(\alpha \equiv$ $\left.N_{f} / 2\right)$ in an interval $[\epsilon, \lambda]$ covering the spectrum of $Q^{\dagger} Q$ :

$$
\lim _{n \rightarrow \infty} P_{n}(x)=x^{-N_{f} / 2} \quad \text { for } \quad x \in[\epsilon, \lambda] .
$$

The inverse determinant of $P_{n}$ in (11) can be represented by $n$ bosonic "pseudofermion" fields. Since for a good approximation a large number $(n=\mathcal{O}(1000))$ of pseudofermion fields would be necessary the direct application of eq. (1) to numerical simulations is not practical. In TSMB a two-step approximation by a product of two polynomials is introduced

$$
\lim _{n_{2} \rightarrow \infty} P_{n_{1}}^{(1)}(x) P_{n_{2}}^{(2)}(x)=x^{-N_{f} / 2} .
$$

The multi-bosonic representation is applied for the first polynomial $P_{n_{1}}^{(1)}$ and the determinant of the second one is taken into account in a stochastic "noisy correction" step. For very small 
fermion masses it is advantageous to perform a final "reweighting correction" in the process of evaluating the expectation values. (For a comprehensive summary of algorithmic details and for references see 顿.)

The DESY-Münster collaboration collected extensive experience on the behaviour of the TSMB algorithm in an application to supersymmetric Yang-Mills (SYM) theory [3 5]. The total amount of computer time used in last year corresponds to $\sim 0.01$ Tflopyear (sustained performance with 64 bit precision). The largest lattice was $12^{3} \cdot 24$. In QCD units obtained from the string tension the spatial extension of this lattice is $\sim 1 \mathrm{fm}$. The statistics was relatively high: several thousands independent gauge configurations per simulation point. Since a Majorana fermion is equivalent to half of a Dirac fermion, the gaugino corresponds to

$\alpha=N_{f} / 2=1 / 4$.

An important factor in the extrapolation to QCD is the dependence on the number of flavours $N_{f}$. It turned out that both execution time of a sweep and autocorrelation of important physical quantities are roughly proportional to the number of pseudofermion fields $n_{1}$. This has to be chosen such that the acceptance rate in the noisy correction step stays high enough. (In our simulations we always had 85-90\% acceptance.) This can be achieved if the deviation norm [2] satisfies

$\delta_{n_{1}}^{2} \leq 5 \cdot 10^{-5}$.

On the basis of this it is possible to estimate the dependence on the number of flavours because, in the limit of a small lower limit of the approximation interval $\epsilon \ll \lambda$, the relation [2]

$\delta_{n}(\alpha ; \epsilon=0, \lambda=1)=\frac{\alpha}{n+1+\alpha} \simeq \frac{N_{f}}{2 n}$

gives a good estimate. This shows that the number of necessary pseudofermion fields $n_{1}$ is approximately proportional to $N_{f}$ and the cost for obtaining a new independent gauge configuration to $N_{f}^{2}$.

Let us note in this context that in a future QCD simulation one would like to have, for instance, a lattice spacing $a \simeq(4 \mathrm{GeV})^{-1}$ and a light quark mass $m_{q} \simeq 4 \mathrm{MeV}$ implying $a m_{q} \simeq 10^{-3}$. This would correspond to $\epsilon \simeq 10^{-6}$, a value which has also been exploited in SYM simulations [3].

Most of the experience on the TSMB algorithm is concentrated up to now on Wilson fermions. The implementation of "moderately improved" actions, as e.g. the clover action of Sheikholeslami-Wohlert, seems possible with reasonable effort. Recently the algorithm has also been applied for staggered fermions [7].

An important question for numerical simulations of odd numbers of quark flavours with Wilson fermions is the effect of the sign of the fermion determinant. In eq. (11) the absolute value of the (real) determinant appears therefore the sign has to be taken into account as a reweighting factor during the evaluation of expectation values. This is a potential problem in at least two respects: the computation of the determinant is only possible on very small lattices and even if the sign of the determinant is known the reweighting with it can in principle cause a serious loss of accuracy due to cancellations. In SYM the problem is similar but the rôle of the determinant is played by the Pfaffian resulting from the Grassmann integration for Majorana fermions.

Concerning the first problem a satisfactory solution is to monitor the sign of the determinant (or Pfaffian) by the spectral flow method [4]. The determinant (or Pfaffian) has a positive sign at small enough hopping parameters (say, $K \leq 0.125)$ and one can count the number of sign changes up to the required (dynamical) hopping parameter by monitoring the flow of the eigenvalues of the hermitean fermion matrix $\tilde{Q} \equiv \gamma_{5} Q$ across zero. The problem of strong cancellations among contributions with opposite sign remains a potential danger.

In SYM with SU(2) gauge group it turned out, however, that the configurations with positive Pfaffian strongly dominate 任. In fact for smaller values of the hopping parameter $K$ no configurations with negative Pfaffian were found. Negative signs at the percent level occurred only for $K$-values beyond $K=K_{0}$ corresponding to zero gaugino mass.

The sign of the determinant in QCD with $\mathbf{2} \oplus \mathbf{1}$ light flavours was up to now not investigated. 
However, the $s$-quark is presumably not light enough for this problem to become serious.

\section{THREE-FLAVOUR QCD}

QCD with three equal mass quarks is not realized in Nature but from the theoretical point of view is rather interesting. An important feature is the possibility of spontaneous CP-violation at negative quark mass predicted long ago by Dashen [8] and Witten [9]. This is a very interesting theoretical issue especially in view of recent and near-future CP-violation experiments in Bfactories. In the lattice literature the question of spontaneous CP- and flavour-symmetry violation has been extensively studied but mainly in case of $N_{f}=2$ flavours [10,11] where it can also be just a lattice artifact [12]. A similar phenomenon also occurs in other models, for instance, in SYM theory with $\mathrm{SU}(3)$ gauge group (see [5]).

Spontaneous CP-violation in QCD with three flavours is predicted by the analysis of the low energy effective chiral Lagrangian

$$
\begin{aligned}
\mathcal{L} & =\frac{f_{\pi}^{2}}{2}\left[\operatorname{Tr}\left(\partial_{\mu} U \partial_{\mu} U^{-1}\right)\right. \\
& \left.+2 \operatorname{Re} \operatorname{Tr}\left(m_{q} e^{-i \theta / 3} U\right)\right] .
\end{aligned}
$$

Here $f_{\pi}$ is the usual parameter of chiral dynamics, $U \in S U(3)$ and $\theta$ is the parameter governing $\mathrm{CP}$-violation. At $\theta \neq 0, \pi$ there is explicit CPviolation whereas at $\theta=3 \pi \equiv \pi$ corresponding to negative quark mass spontaneous $\mathrm{CP}$-violation is expected. The $\theta$-dependence of the energies of different ground states are illustrated by fig. 1.

This picture is the solution of the puzzle emphasized in [9]: the vacuum energy in the $N_{c} \rightarrow$ $\infty$ limit is

$E(\theta)=N_{c}^{2} h\left(\frac{\theta}{N_{c}}\right)=E(\theta+2 \pi)$.

The solution can only be a multi-branched function

$E(\theta)=N_{c}^{2} \min _{k} h\left(\frac{\theta+2 k \pi}{N_{c}}\right)$.

Numerical simulations could prove this picture following from low energy effective chiral Lagrangians.

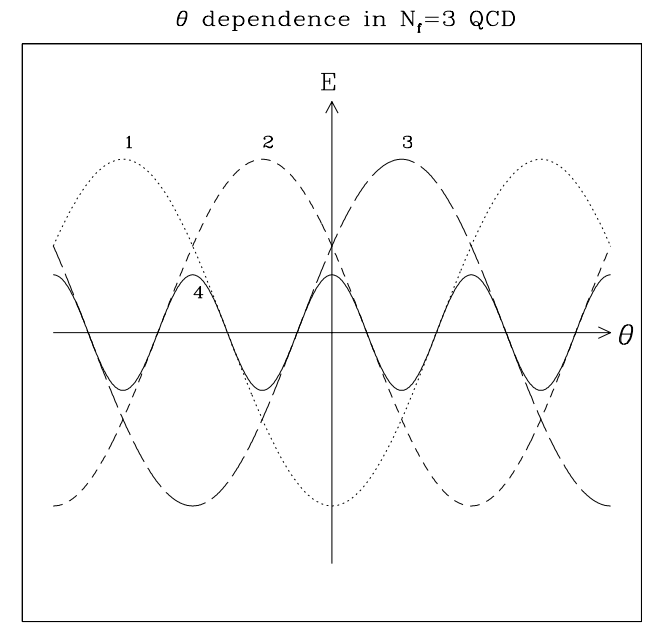

Figure 1. The energies of different branches of ground states as a function of the $\theta$-parameter.

Acknowledgement: It is a pleasure to thank the members of the DESY-Münster collaboration for helpful discussions.

\section{REFERENCES}

1. I. Montvay, Nucl. Phys. B466 (1996) 259.

2. I. Montvay, Comput. Phys. Commun. 109 (1998) 144.

3. R. Kirchner, S. Luckmann, I. Montvay, K. Spanderen, J. Westphalen, Phys. Lett. B446 (1999) 209.

4. I. Campos, A. Feo, R. Kirchner, S. Luckmann, I. Montvay, G. Münster, K. Spanderen, J. Westphalen, hep-lat/9903014, to appear in Eur. Phys. J. C.

5. A. Feo, R. Kirchner, S. Luckmann, I. Montvay, G. Münster, A. Vladikas, this Proceedings.

6. M. Lüscher, Nucl. Phys. B418 (1994) 637.

7. S. Hands, I. Montvay, S. Morrison, M. Oevers, J. Skullerud, in preparation.

8. R. Dashen, Phys. Rev. D3 (1971) 1879.

9. E. Witten, Annals of Phys. 128 (1980) 363.

10. S. Aoki, Phys. Rev. D30 (1984) 2653;

Phys. Rev. Lett. 57 (1986) 3136.

11. M. Creutz, Phys. Rev. D52 (1995) 2951.

12. S. Sharpe, R. Singleton, Phys. Rev. D58 (1998) 074501. 